\title{
Analytical-numerical localization of hidden attractor in electrical Chua's circuit
}

Kuznetsov N., Kuznetsova O., Leonov G., Vagaitsev V.

Draft $^{1} 2$

Abstract. Study of hidden oscillations and hidden chaotic attractors (basin of attraction of which does not contain neighborhoods of equilibria) requires the development of special analytical-numerical methods. Development and application of such methods for localization of hidden chaotic attractors in dynamical model of Chua's circuit with nonlinearity sign are demonstrated in this work.

Keywords: chaotic hidden attractor, smooth Chua system, Chua circuits, hidden oscillation, describing function method

\section{Introduction}

The classical attractors of Lorenz [1], Rossler [2], Chua [3], Chen [4], and other widely-known attractors are those excited from unstable equilibria (self excited attractors). From computational point of view this allows one to use standard numerical method, in which after transient process a trajectory, started from a point of unstable manifold in the neighborhood of equilibrium, reaches an attractor and identifies it.

However there are attractors of another type: hidden attractors, a basin of attraction of which does not contain neighborhoods of equilibria [6]. Here equilibria are not "connected" with attractor and creation of numerical procedure of integration of trajectories for the passage from equilibrium to attractor is impossible because the neighbourhood of equilibrium does not belong to such attractor. The simplest examples of systems with such hidden attractors are hidden oscillations in counterexamples to widely-known Aizerman's and Kalman's conjectures on absolute stability (see, e.g., $[8,9,10]$ ). Similar computational problems arise in investigation of semi-stable and nested limit cycles in 16th Hilbert problem (see, e.g., $\mid 11,12,13\rfloor)$.

In 2010, for the first time, a chaotic hidden attractor [5] was computed in generalized Chua's circuit (which can be used for hidden chaotic communication [17]) and then one was discovered in classical Chua's circuit.

Further a special analytical-numerical algorithm for localization of hidden attractors is considered.

Chua's circuit (see Fig. 1) can be described by differential equations in dimensionless coordinates:

$$
\begin{aligned}
& \dot{x}=\alpha(y-x)-\alpha f(x), \\
& \dot{y}=x-y+z, \\
& \dot{z}=-(\beta y+\gamma z) .
\end{aligned}
$$

Here the function

$$
\begin{array}{r}
f(x)=m_{1} x+\left(m_{0}-m_{1}\right) \operatorname{sat}(x)= \\
=m_{1} x+\frac{1}{2}\left(m_{0}-m_{1}\right)(|x+1|-|x-1|)
\end{array}
$$

characterizes a nonlinear element, of the system, called Chua's diode; $\alpha, \beta, \gamma, m_{0}, m_{1}$ are parameters of the system. In this system it was discovered the strange attractors $\lfloor 14,15\rfloor$ called then Chua's attractors. All

\footnotetext{
${ }^{1}$ Nikolay V. Kuznetsov, nkuznetsov239 at gmail.com (correspondence author)

${ }^{2} \mathrm{PDF}$ slides http://www.math.spbu.ru/user/nk/PDF/Hidden-attractor-localization-Chua-circuit.pdf
} 


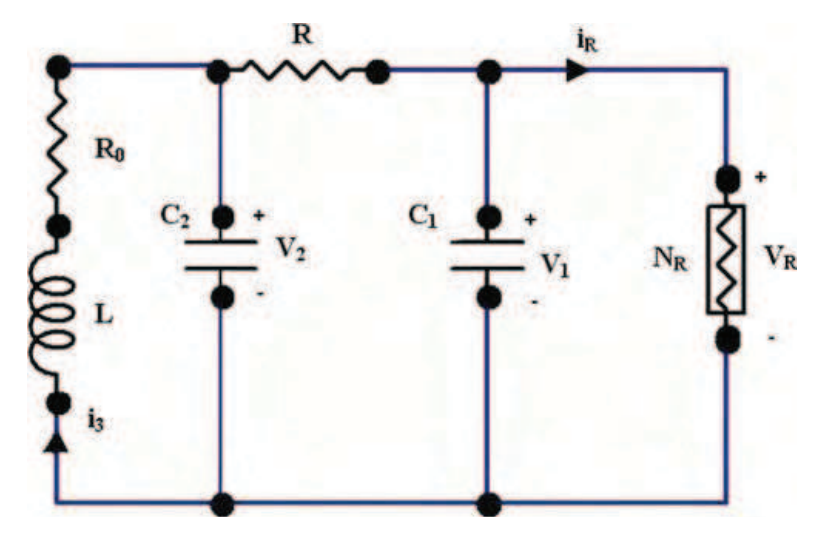

Figure 1: Classical Chua's circuit

known classical Chua's attractors are the attractors that are excited from unstable equilibria and this makes it possible to compute such attractors with relative easy (see, e.g., attractors gallery in [16]).

The applied in this work algorithm shows the possibility of existence of hidden attractor in system (1). Note that L. Chua himself, analyzing in the work [3] different cases of attractor existence in Chua's circuit, does not admit the existence of such hidden attractor.

\section{Analytical-numerical method for hidden attractors localization}

For numerical location of hidden oscillations it is turns out to be effective methods based on homotopy where a sequence of similar systems is consider such that initial data for numerical localization of periodic solution (starting periodic solution) in the first starting system can be obtained analytically and then transformation of this starting periodic solution in the transition from one system to another is followed numerically.

Consider a system

$$
\frac{d \mathbf{x}}{d t}=\mathbf{P x}+\boldsymbol{\psi}(\mathbf{x}), \mathbf{x} \in \mathbb{R}^{n},
$$

where $\mathbf{P}$ is a constant $n \times n$-matrix, $\boldsymbol{\psi}(\mathbf{x})$ is a continuous vector-function, and $\boldsymbol{\psi}(0)=0$.

Define a matrix $\mathbf{K}$ in such a way that the matrix

$$
\mathbf{P}_{0}=\mathbf{P}+\mathbf{K}
$$

has a pair of purely imaginary eigenvalues $\pm i \omega_{0}\left(\omega_{0}>0\right)$ and the rest of its eigenvalues have negative real parts. We assume that such $\mathbf{K}$ exists. Rewrite system (3) as

$$
\frac{d \mathbf{x}}{d t}=\mathbf{P}_{0} \mathbf{x}+\boldsymbol{\varphi}(\mathbf{x}),
$$

where $\varphi(\mathrm{x})=\psi(\mathrm{x})-\mathbf{K x}$.

Introduce a finite sequence of functions $\varphi^{0}(\mathrm{x}), \varphi^{1}(\mathrm{x}), \ldots, \varphi^{m}(\mathrm{x})$ such that the graphs of neighboring functions $\varphi^{j}(\mathrm{x})$ and $\varphi^{j+1}(\mathrm{x})$ slightly differ from one another, the function $\varphi^{0}(\mathrm{x})$ is small, and $\varphi^{m}(\mathrm{x})=\varphi(\mathrm{x})$. Using a smallness of function $\varphi^{0}(\mathbf{x})$, we can apply and mathematically strictly justify $[7,8]$ the method of harmonic linearization (describing function method) for the system

$$
\frac{d \mathbf{x}}{d t}=\mathbf{P}_{0} \mathbf{x}+\varphi^{0}(\mathbf{x}),
$$

and determine a stable nontrivial periodic solution $\mathbf{x}^{0}(t)$. For the localization of attractor of original system (5), we shall follow numerically the transformation of this periodic solution (a starting oscillating attractor - 
an attractor, not including equilibria, denoted further by $\mathcal{A}_{0}$ ) with increasing $j$. Here two cases are possible: all the points of $\mathcal{A}_{0}$ are in an attraction domain of attractor $\mathcal{A}_{1}$, being an oscillating attractor of the system

$$
\frac{d \mathbf{x}}{d t}=\mathbf{P}_{0} \mathbf{x}+\varphi^{j}(\mathbf{x})
$$

with $j=1$, or in the change from system (6) to system (7) with $j=1$ it is observed a loss of stability bifurcation and the vanishing of $\mathcal{A}_{0}$. In the first case the solution $\mathbf{x}^{1}(t)$ can be determined numerically by starting a trajectory of system (7) with $j=1$ from the initial point $\mathbf{x}^{0}(0)$. If in the process of computation the solution $\mathbf{x}^{1}(t)$ has not fallen to an equilibrium and it is not increased indefinitely (here a sufficiently large computational interval $[0, T]$ should always be considered), then this solution reaches an attractor $\mathcal{A}_{1}$. Then it is possible to proceed to system (7) with $j=2$ and to perform a similar procedure of computation of $\mathcal{A}_{2}$, by starting a trajectory of system (7) with $j=2$ from the initial point $\mathbf{x}^{1}(T)$ and computing the trajectory $\mathbf{x}^{2}(t)$.

Proceeding this procedure and sequentially increasing $j$ and computing $\mathbf{x}^{j}(t)$ (being a trajectory of system (7) with initial data $\mathbf{x}^{j-1}(T)$ ) we either arrive at the computation of $\mathcal{A}_{m}$ (being an attractor of system (7) with $j=m$, i.e. original system (5)), or, at a certain step, observe a loss of stability bifurcation and the vanishing of attractor.

To determine the initial data $\mathbf{x}^{0}(0)$ of starting periodic solution, system (6) with nonlinearity $\varphi^{0}(\mathbf{x})$ can be transformed by linear nonsingular transformation $\mathbf{S}$ to the form

$$
\begin{aligned}
& \dot{y}_{1}=-\omega_{0} y_{2}+\varepsilon \varphi_{1}\left(y_{1}, y_{2}, \mathbf{y}_{3}\right), \\
& \dot{y}_{2}=\omega_{0} y_{1}+\varepsilon \varphi_{2}\left(y_{1}, y_{2}, \mathbf{y}_{3}\right), \\
& \dot{\mathbf{y}}_{3}=\mathbf{A}_{\mathbf{3}} \mathbf{y}_{3}+\varepsilon \boldsymbol{\varphi}_{3}\left(y_{1}, y_{2}, \mathbf{y}_{3}\right)
\end{aligned}
$$

Here $\mathbf{A}_{\mathbf{3}}$ is a constant $(n-2) \times(n-2)$ matrix, all eigenvalues of which have negative real parts, $\boldsymbol{\varphi}_{3}$ is an $(n-2)$-dimensional vector-function, $\varphi_{1}, \varphi_{2}$ are certain scalar functions. Without loss of generality, it may be assumed that for the matrix $\mathbf{A}_{\mathbf{3}}$ there exists positive number $\alpha>0$ such that

$$
\mathbf{x}_{3}^{*}\left(\mathbf{A}_{\mathbf{3}}+\mathbf{A}_{\mathbf{3}}{ }^{*}\right) \mathbf{x}_{3} \leq-2 \alpha\left|\mathbf{x}_{3}\right|^{2}, \quad \forall \mathbf{x}_{3} \in \mathbb{R}^{n-2}
$$

Introduce the following describing function

$$
\begin{aligned}
& \Phi(a)=\int_{0}^{2 \pi / \omega_{0}}\left[\varphi_{1}\left(\left(\cos \omega_{0} t\right) a,\left(\sin \omega_{0} t\right) a, 0\right) \cos \omega_{0} t+\right. \\
& \left.+\varphi_{2}\left(\left(\cos \omega_{0} t\right) a,\left(\sin \omega_{0} t\right) a, 0\right) \sin \omega_{0} t\right] d t
\end{aligned}
$$

Theorem 1 in] If it can be found a positive $a_{0}$ such that

$$
\Phi\left(a_{0}\right)=0
$$

and $\Phi^{\prime}\left(a_{0}\right)<0$ then there is a periodic solution in system (6) with the initial data $\mathbf{x}^{0}(0)=\mathbf{S}\left(y_{1}(0), y_{2}(0), \mathbf{y}_{3}(0)\right)^{*}$

$$
y_{1}(0)=a_{0}+O(\varepsilon), y_{2}(0)=0, \mathbf{y}_{3}(0)=\mathbf{O}_{\mathbf{n}-\mathbf{2}}(\varepsilon) .
$$

Here $\mathbf{O}_{\mathbf{n}-\mathbf{2}}(\varepsilon)$ is an $(n-2)$-dimensional vector such that all its components are $O(\varepsilon)$. 


\section{Localization of hidden attractor in Chua's system}

We now apply the above algorithm to analysis of Chua's system with scalar nonlinearity. For this purpose, rewrite Chua's system (1) in the form (3)

$$
\frac{d \mathbf{x}}{d t}=\mathbf{P} \mathbf{x}+\mathbf{q} \psi\left(\mathbf{r}^{*} \mathbf{x}\right), \quad \mathbf{x} \in \mathbb{R}^{3} .
$$

Here

$$
\begin{aligned}
& \mathbf{P}, \mathbf{q}, \mathbf{r}=\left(\begin{array}{ccc}
-\alpha\left(m_{1}+1\right) & \alpha & 0 \\
1 & -1 & 1 \\
0 & -\beta & -\gamma
\end{array}\right),\left(\begin{array}{c}
-\alpha \\
0 \\
0
\end{array}\right),\left(\begin{array}{l}
1 \\
0 \\
0
\end{array}\right), \\
& \psi(\sigma)=\left(m_{0}-m_{1}\right) \operatorname{sat}(\sigma) .
\end{aligned}
$$

Introduce the coefficient $k$ and small parameter $\varepsilon$, and represent system (12) as (6)

$$
\frac{d \mathbf{x}}{d t}=\mathbf{P}_{\mathbf{0}} \mathbf{x}+\mathbf{q} \varepsilon \varphi\left(\mathbf{r}^{*} \mathbf{x}\right)
$$

where

$$
\begin{aligned}
& \mathbf{P}_{\mathbf{0}}=\mathbf{P}+k \mathbf{q} \mathbf{r}^{*}=\left(\begin{array}{ccc}
-\alpha\left(m_{1}+1+k\right) & \alpha & 0 \\
1 & -1 & 1 \\
0 & -\beta & -\gamma
\end{array}\right), \\
& \lambda_{1,2}^{\mathbf{P}_{0}}= \pm i \omega_{0}, \lambda_{3}^{\mathbf{P}_{0}}=-d, \\
& \varphi(\sigma)=\psi(\sigma)-k \sigma=\left(m_{0}-m_{1}\right) \operatorname{sat}(\sigma)-k \sigma .
\end{aligned}
$$

In practice, to determine $k$ and $\omega_{0}$ it is used the transfer function $W(p)$ of system $(3)$ :

$$
W_{\mathbf{P}_{\mathbf{0}}}(p)=\mathbf{r}^{*}(\mathbf{P}-p \mathbf{I})^{-1} \mathbf{q},
$$

where $p$ is a complex variable. Then $\operatorname{Im} W\left(i \omega_{0}\right)=0$ and $k$ is computed then by formula $k=-\left(\operatorname{Re} W\left(i \omega_{0}\right)\right)^{-1}$.

By nonsingular linear transformation $\mathbf{x}=$ Sy system (13) can be reduced to the form

$$
\frac{d \mathbf{y}}{d t}=\mathbf{A y}+\mathbf{b} \varepsilon \varphi\left(\mathbf{c}^{*} \mathbf{y}\right)
$$

where

$$
\mathbf{A}, \mathbf{b}, \mathbf{c}=\left(\begin{array}{ccc}
0 & -\omega_{0} & 0 \\
\omega_{0} & 0 & 0 \\
0 & 0 & -d
\end{array}\right),\left(\begin{array}{c}
b_{1} \\
b_{2} \\
1
\end{array}\right),\left(\begin{array}{c}
1 \\
0 \\
-h
\end{array}\right) .
$$

Further, using the equality of transfer functions of systems (13) and (14), we obtain

$$
W_{\mathbf{A}}(p)=\mathbf{r}^{*}\left(\mathbf{P}_{0}-p \mathbf{I}\right)^{-1} \mathbf{q}
$$

This implies the following relations

$$
\begin{aligned}
& k=\frac{-\alpha\left(m_{1}+m_{1} \gamma+\gamma\right)+\omega_{0}^{2}-\gamma-\beta}{\alpha(1+\gamma)}, \\
& d=\frac{\alpha+\omega_{0}^{2}-\beta+1+\gamma+\gamma^{2}}{1+\gamma} \\
& h=\frac{\alpha\left(\gamma+\beta-(1+\gamma) d+d^{2}\right)}{\omega_{0}^{2}+d^{2}} \\
& b_{1}=\frac{\alpha\left(\gamma+\beta-\omega_{0}^{2}-(1+\gamma) d\right)}{\omega_{0}^{2}+d^{2}} \\
& b_{2}=\frac{\alpha\left((1+\gamma-d) \omega_{0}^{2}+(\gamma+\beta) d\right)}{\omega_{0}\left(\omega_{0}^{2}+d^{2}\right)} .
\end{aligned}
$$


System (13) can be reduced to the form (14) by the nonsingular linear transformation $\mathbf{x}=\mathbf{S y}$. Having solved the following matrix equations

$$
\mathbf{A}=\mathbf{S}^{-1} \mathbf{P}_{0} \mathbf{S}, \quad \mathbf{b}=\mathbf{S}^{-1} \mathbf{q}, \quad \mathbf{c}^{*}=\mathbf{r}^{*} \mathbf{S},
$$

one can obtain the transformation matrix

$$
\mathbf{S}=\left(\begin{array}{lll}
s_{11} & s_{12} & s_{13} \\
s_{21} & s_{22} & s_{23} \\
s_{31} & s_{32} & s_{33}
\end{array}\right)
$$

By (11), for small enough $\varepsilon$ we determine initial data for the first step of multistage localization procedure

$$
\mathbf{x}(0)=\mathbf{S y}(0)=\mathbf{S}\left(\begin{array}{c}
a_{0} \\
0 \\
0
\end{array}\right)=\left(\begin{array}{c}
a_{0} s_{11} \\
a_{0} s_{21} \\
a_{0} s_{31}
\end{array}\right) \text {. }
$$

Returning to Chua's system denotations, for determining the initial data of starting solution of multistage procedure we have the following formulas

$$
\begin{array}{r}
x(0)=a_{0}, y(0)=a_{0}\left(m_{1}+1+k\right), \\
z(0)=a_{0} \frac{\alpha\left(m_{1}+k\right)-\omega_{0}^{2}}{\alpha} .
\end{array}
$$

Consider system (13) with the parameters

$$
\begin{array}{r}
\alpha=8.4562, \beta=12.0732, \gamma=0.0052, \\
m_{0}=-0.1768, m_{1}=-1.1468 .
\end{array}
$$

Note that for the considered values of parameters there are three equilibria in the system: a locally stable zero equilibrium and two saddle equilibria.

Now we apply the above procedure of hidden attractors localization to Chua's system (12) with parameters (18). For this purpose, compute a starting frequency and a coefficient of harmonic linearization. We have

$$
\omega_{0}=2.0392, \quad k=0.2098 \text {. }
$$

Then, compute solutions of system (13) with nonlinearity $\varepsilon \varphi(x)=\varepsilon(\psi(x)-k x)$, sequentially increasing $\varepsilon$ from the value $\varepsilon_{1}=0.1$ to $\varepsilon_{10}=1$ with the step 0.1 .

By (15) and (17) we obtain the initial data

$$
x(0)=9.4287, y(0)=0.5945, z(0)=-13.4705
$$

for the first step of multistage procedure for the construction of solutions. For the value of parameter $\varepsilon_{1}=0.1$, after transient process the computational procedure reaches the starting oscillation $\mathbf{x}^{1}(t)$. Further, by the sequential transformation $\mathbf{x}^{j}(t)$ with increasing the parameter $\varepsilon_{j}$, using the numerical procedure, for original Chua's system (12) the set $\mathcal{A}_{\text {hidden }}$ is computed. This set is shown in Fig. 3.

The considered system has three stationary points: the stable zero point $F_{0}$ and the symmetric saddles $S_{1}$ and $S_{2}$. To zero equilibrium $F_{0}$ correspond the eigenvalues $\lambda_{1}^{F_{0}}=-7.9591$ and $\lambda_{2,3}^{F_{0}}=-0.0038 \pm 3.2495 i$ and to the saddles $S_{1}$ and $S_{2}$ correspond the eigenvalues $\lambda_{1}^{S_{1,2}}=2.2189$ and $\lambda_{2,3}^{S_{1,2}}=-0.9915 \pm 2.4066 i$. The behavior of trajectories of system in a neighborhood of equilibria is shown in Fig. 3 . 


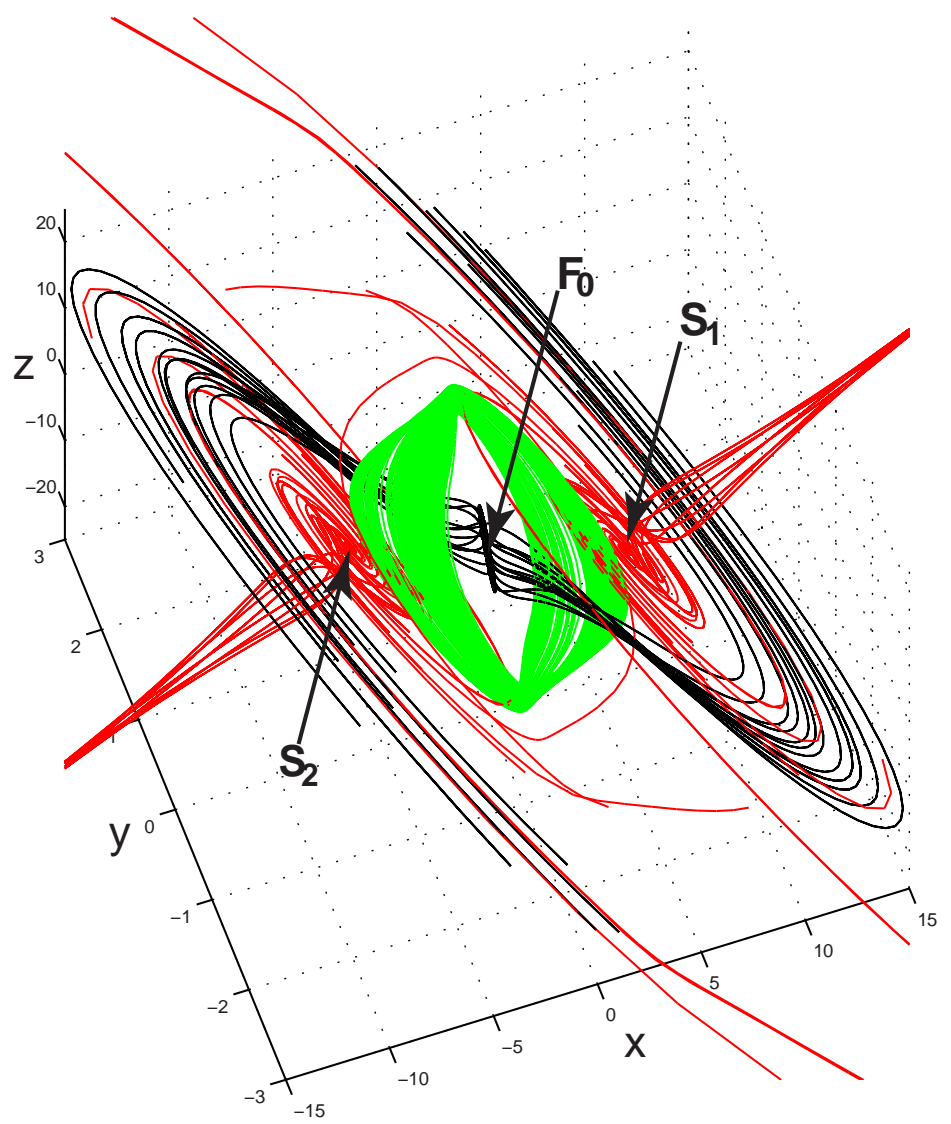

Figure 2: Equilibrium, stable manifolds of saddles, and localization of hidden attractor.

Figure 3: Hidden attractor projections on $(x, y),(x, z)$, and $(y, z)$ 
Figure 4: Projection of the solutions of the modified Chua's system on the plane $(x, y)$ for $i=3$. Nonlinearity $\theta^{i}(x)$ and stability sectors.

Figure 5: Projection of the solutions of the modified Chua's system on the plane $(x, y)$ for $i=5$. Nonlinearity $\theta^{i}(x)$ and stability sectors.

We remark that here positive Lyapunov exponent $[18]^{3}$. corresponds to the computed trajectories.

By the above and with provision for the remark on the existence, in system, of locally stable zero equilibrium and two saddle equilibria (trajectories from the neighborhood of these saddles tend to zero or to infinity), we arrive at the conclusion that in $\mathcal{A}_{\text {hidden }}$ a hidden strange attractor is computed.

Now let us consider localization of hidden oscillation in the Chua's circuit with modified nonlinear characteristic - discontinuous nonlinearity $\operatorname{sign}(x)$ instead of sat $(x)$. For this we consider the system (12) with nonlinearity of special form

$$
\theta^{i}(x)=\psi(x)+\frac{i}{n}\left(\left(m_{0}-m_{1}\right) \operatorname{sign}(x)-\psi(x)\right), \quad i=1, \ldots, n
$$

and apply the same numerical procedure to the new system with $n=10$, increasing the value of the parameter $i$ from 1 up to 10 . Projections of the solutions of the system (12) with nonlinearity (19) on the plane $(x, y)$ for $i=3,5,7,10$ are shown in Figs. 4-7 respectively.

\section{Conclusions}

In the present work the application of special analytical-numerical algorithm for hidden attractor localization is discussed. The existence of such hidden attractors in classical and modified Chua's circuits is demonstrated.

It is also can be noted that to obtain existence of hidden attractor in Chua's circuit one can artificially modify $\lfloor 20,19,7\rfloor$ diode characteristics to stabilize zero stationary point by inserting small stable zone around zero stationary point into nonlinearity.

\section{Acknowledgments.}

This work was partly supported by grants from Academy of Finland, Ministry of Education and Science (Russia), RFBR, and Saint-Petersburg State University.

\footnotetext{
${ }^{3}$ Lyapunov exponents (LEs) were introduced by Lyapunov for the analysis of stability by the first approximation for regular time-varying linearizations, where negativeness of the largest Lyapunov exponent indicated stability. While there are known. Perron effects $\mid 18$. of the largest Lyapunov exponent sign inversions for non regular time-varying linearizations, computation of Lyapunov exponents for linearization of nonlinear autonomous system along non stationary trajectories is widely used for investigation of chaos, where positiveness of the largest Lyapunov exponent is often considered as indication of chaotic behavior in considered nonlinear system.
}

Figure 6: Projection of the solutions of the modified Chua's system on the plane $(x, y)$ for $i=7$. Nonlinearity $\theta^{i}(x)$ and stability sectors. 
Figure 7: Projection of the solutions of the modified Chua's system on the plane $(x, y)$ for $i=10$. Nonlinearity $\theta^{i}(x)$ and stability sectors.

\section{References}

[1] Lorenz, E.N.: Deterministic nonperiodic flow. J. Atmos. Sci. 20, 130-141 (1963)

[2] Rossler, O.E.: An Equation for Continuous Chaos. Physics Letters. 57A(5), 397-398 (1976)

[3] Chua, L.O., Lin, G.N.: Canonical Realization of Chua's Circuit Family. IEEE Transactions on Circuits and Systems. 37(4), 885-902 (1990)

[4] Chen, G., Ueta, T.: Yet another chaotic attractor. Int. J. Bifurcation and Chaos. 9, 1465-1466 (1999)

[5] Kuznetsov N.V., Leonov G.A., Vagaitsev V.I. Analytical-numerical method for attractor localization of generalized Chua's system // IFAC Proceedings Volumes (IFAC-PapersOnline). 2010. 4(1). (doi:10.3182/20100826-3-TR-4016.00009)

[6] Leonov, G.A., Kuznetsov, N.V., Vagaytsev, V.I.: Localization of hidden Chua's attractors. Physics Letters A. 375(35), 2230-2233 (2011) [doi:10.1016/j.physleta.2011.04.037]

[7] Leonov, G.A., Vagaitsev, V.I., Kuznetsov, N.V.: Algorithm for localizing Chua attractors based on the harmonic linearization method. Doklady Mathematics. 82(1), 663-666 (2010)

[8] Leonov, G.A., Bragin, V.O., Kuznetsov, N.V.: Algorithm for Constructing Counterexamples to the Kalman Problem. Doklady Mathematics. 82(1), 540-542 (2010)

[9] Leonov G.A., Kuznetsov N.V. Algorithms for Searching for Hidden Oscillations in the Aizerman and Kalman Problems // Doklady Mathematics. 8(1). 475-481 (2011) (doi:10.1134/S1064562411040120)

[10] Bragin V.O., Kuznetsov N.V., Leonov G.A., Vagaitsev V.I. Algorithms for Finding Hidden Oscillations in Nonlinear, Systems. The Aizerman and Kalman Conjectures and Chua's Circuits // Journal of Computer and Systems Sciences International. 50(4). 511-544 (2011). (doi:10.1134/S106423071104006X)

[11] Kuznetsov N.V., Leonov G.A. Lyapunov quantities, limit cycles and strange behavior of trajectories in two-dimensional quadratic systems. Journal of Vibroengineering. 10(4), 460-467 (2008)

[12] Leonov, G.A., Kuznetsov, N.V.: Limit cycles of quadratic systems with a perturbed weak focus of order 3 and a saddle equilibrium at infinity. Doklady Mathematics. 82(2), 693-696 (2010)

[13] Leonov, G.A., Kuznetsov, N.V., Kudryashova, E.V.: A Direct Method for Calculating Lyapunov Quantities of Two-Dimensional Dynamical Systems. Proceedings of the Steklov Institute Of Mathematics. 272(SUPPL. 1), S119-S127 (2011)

[14] Chua, L.O.: A Zoo of Strange Attractors from the Canonical Chua's Circuits. Proceedings of the IEEE 35th Midwest Symposium on Circuits and Systems. 2, 916-926 (1992)

[15] Chua, L.O.: A Glimpse of Nonlinear Phenomena from Chua's Oscillator. Philosophical Transactions: Physical Sciences and Engineering. 353, 3-12 (1995)

[16] Bilotta, E., Pantano, P.: A gallery of Chua attractors. World scientific series on nonlinear science (2008)

[17] Shi, Z., Hong, S., Chen, K.: Experimental study on tracking the state of analog Chua's circuit with particle filter for chaos synchronization. Physics Letters A. 372(34), 5575-5580 (2008) 
[18] Leonov, G.A., Kuznetsov, N.V.: Time-Varying Linearization and the Perron effects. International Journal of Bifurcation and Chaos. 17(4), 1079-1107 (2007)

[19] Savaci, F.A., Gunel, S.: Harmonic Balance Analysis of the Generalized Chua's Circuit. International Journal of Bifurcation and Chaos. 16(8), 2325-2332 (2006)

[20] Suykens, J.A.K., Huang, A., Chua, L.O.: A Family of n-scroll Attractors from a Generalized Chuas Circuit. AEU-International Journal of Electronics \& Communications. 51(3), 131-138 (1997) 